ESAIM: PROCEEDINGS, October 2007, Vol. 22, 1-14

Gabriel Caloz \& Monique Dauge, Editors

\title{
ACOUSTIC PROPAGATION IN A FLOW: NUMERICAL SIMULATION OF THE TIME-HARMONIC REGIME
}

\author{
Anne-Sophie Bonnet-Ben Dhia ${ }^{1,2}$, Eve-Marie Duclairoir ${ }^{1}$ and Jean-Francois \\ MERCIER $^{1}$
}

\begin{abstract}
We consider the time-harmonic acoustic radiation of a source in a moving fluid. The problem is set in a two-dimensional infinite duct and the mean flow is a subsonic parallel shear flow, with a regular profile. We deal with an equation (due to Galbrun) whose unknown is the displacement perturbation. We show how to solve the problem with a finite element method by writing a "regularized" or "augmented" formulation and using Perfectly Matched Layers to select the outgoing solution. Due to the presence of a non-local term coming from the regularization, an iterative process of resolution is preferred, which converges faster for weaker shear. Some mathematical results are established in the dissipative case. Numerical illustrations are finally presented.
\end{abstract}

\section{INTRODUCTION}

The influence of a flow on the acoustic propagation still raises several open questions although it is involved in many practical applications : in particular a more efficient numerical simulation of acoustic propagation would be a useful tool to improve noise reducing in planes or cars industry.

Our aim is to compute by a finite element method the acoustic field radiated by a source, in a fluid in flow, in time-harmonic regime $\left(e^{-i \omega t}\right)$ and in an unbounded domain. The unknown is a small perturbation of a given flow, which naturally leads to consider linearized equations. Contrary to the classical case of acoustics in a fluid at rest, the obtained problem is vectorial, since the presence of a mean flow generally couples acoustics and hydrodynamics.

Only in the case of a potential flow, acoustic perturbations are modelized by a scalar generalized Helmholtz equation : in particular, if the flow is uniform far from the source, the problem can be solved in a classical way, by coupling finite elements with integral [1] or modal [2] representation of the far-field.

For a non-potential flow, most of the works concern a resolution of Euler's linearized equations in the time domain : Euler's system is a first order system involving the velocity and pressure perturbations, which can be solved by using finite difference methods [3-8] or discontinuous Galerkin methods $[9,10]$.

Our choice is different and it seems to us more adapted for solving the time harmonic problem with finite elements. The equation we consider is due to Galbrun [11]. It is a second order system involving the displacement perturbation.

\footnotetext{
${ }^{1}$ Laboratoire POEMS, UMR 2706, CNRS-ENSTA-INRIA, 32 Boulevard Victor, 75015 Paris, FRANCE;

e-mail: Anne-Sophie.Bonnet-Bendhia@ensta.fr \& Eve-Marie.Duclairoir@ensta.fr \& Jean-Francois.Mercier@ensta.fr

2 CERFACS, 42, Avenue Gaspard Coriolis, 31057 Toulouse Cedex 01, France
} 
Although Galbrun's equation is very similar to classical wave equations, the direct use of a finite element method to solve it leads to very bad results. Also the treatment of unbounded domains has not been satisfactorily taken into account up to now, the difficulty being to handle simultaneously acoustic and hydrodynamic phenomena. In particular, convection of vortices requires different treatments upstream and downstream, which is not tractable with a variational approach.

Introducing the pressure as a new unknown, a mixed finite element scheme has been developed [12,13], which has been checked to be stable in several applications. However this mixed approach does not help to deal satisfactorily with the convection of vortices.

We propose here a different approach, based on the so-called regularization of Galbrun's equation. Both difficulties mentioned above are then solved : the stability of a classical nodal finite element scheme is ensured and the hydrodynamic phenomenon is taken explicitly into account, allowing to introduce in the PMLs (Perfectly Matched Layers) the specific upstream-downstream behavior of the solution.

\section{The Physical PRoblem POSED IN A WAVEGUide}

A two-dimensional problem set in an infinite duct $\Omega=\{-\infty<x<+\infty, 0<y<l\}$ whose boundaries are rigid is considered. This duct is filled with a compressible fluid in parallel and subsonic flow : the flow is characterized by the Mach number profile, $-1<M(y)<1$.

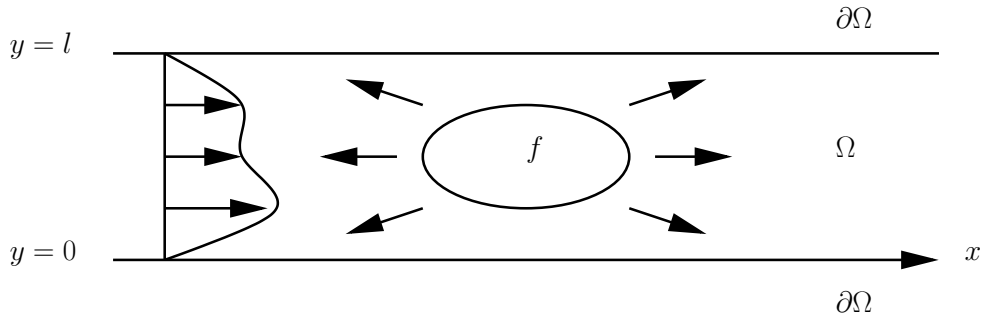

In time-harmonic regime, the displacement perturbation is supposed to read

$$
\Re e\left(\boldsymbol{u}(x) e^{-i \omega t}\right), \quad \omega>0
$$

and we look for solving a problem of the following form (where $f$ is a source supposed to be compactly supported in the duct):

$$
\begin{array}{ll}
\mathrm{D}^{2} \boldsymbol{u}-\boldsymbol{\nabla}(\operatorname{div} \boldsymbol{u})=\boldsymbol{f} & \text { in } \Omega, \\
\boldsymbol{u} \cdot \boldsymbol{n}=0 & \text { on } \partial \Omega,
\end{array}
$$

where we have introduced $\mathrm{D}$ the convective derivative operator defined by $\mathrm{D}=-i k+M \partial / \partial x(k=\omega / c$ is the wave number, $c$ the sound speed).

The problem must be closed with radiation conditions at infinity, expressing the fact that the outgoing solution is sought. These conditions can be expressed thanks to the duct modes, but their numerical treatment is delicate, in particular because of the existence of a hydrodynamic modes continuum, modes convected by the flow. The combined use of a regularized formulation and of PMLs will allow us to avoid this difficulty.

The uniform flow case helps to better understand the phenomena modelized by Galbrun's equation. Indeed, if $M$ is constant, it is easy to check that the acoustic pressure $p=-\operatorname{div} \boldsymbol{u}$ and the vorticity $\psi=$ curl $\boldsymbol{u}$ both satisfy two uncoupled problems: $p$ is solution of the convected wave equation

$$
\mathrm{D}^{2} p-\Delta p=-\operatorname{div} \boldsymbol{f}
$$

and $\psi$ is solution of the equation

$$
\mathrm{D}^{2} \psi=\operatorname{curl} \boldsymbol{f}
$$

that modelizes the convection of vortices by the flow. In the non-uniform flow case, these two phenomena are coupled. 
Finally, our aim is to calculate the outgoing solution of (1) using finite elements and PMLs.

\section{REgUlarized FORMULATION}

Let us suppose for the moment that we try to approach by finite elements the solution of Galbrun's equation in a bounded set (a part $\Omega_{b}$ of the duct $\Omega$ ).

\subsection{Without flow}

In the absence of flow $(M=0)$, it is known that Galbrun's equation

$$
-\nabla(\operatorname{div} \boldsymbol{u})-k^{2} \boldsymbol{u}=\boldsymbol{f},
$$

can not be discretized thanks to Lagrange finite elements. This is due to a lack of compacity (of the injection from $H_{d i v}$ into $\mathrm{L}^{2}$ ), difficulty entirely similar to the one widely studied in the framework of harmonic Maxwell equations. The solutions to this problem developed in the literature consist in using Raviart-Thomas elements, $H_{d i v}$-conforming, or in writing an equivalent regularized formulation of the problem. This is achieved by noting, after having taken the curl of Galbrun's equation, that the curl of the solution can be calculated a priori:

$$
\operatorname{curl} \boldsymbol{u}=-\frac{1}{k^{2}} \operatorname{curl} \boldsymbol{f} .
$$

We deduce that $\boldsymbol{u}$ is solution of the following equation, where $\psi_{f}=-\operatorname{curl} \boldsymbol{f} / k^{2}$ is a data:

$$
-\boldsymbol{\nabla}(\operatorname{div} \boldsymbol{u})+\operatorname{curl}\left(\operatorname{curl} \boldsymbol{u}-\psi_{f}\right)-k^{2} \boldsymbol{u}=\boldsymbol{f},
$$

which can be written:

$$
-\Delta \boldsymbol{u}-k^{2} \boldsymbol{u}=\operatorname{curl} \psi_{f}+\boldsymbol{f} .
$$

Then this equation can be discretized thanks to classical Lagrange finite elements. If the domain has no reentrant corner, the solution of the latter problem is the good solution of (1) (the one satisfying curl $\boldsymbol{u}=\psi_{f}$ ).

\subsection{With a flow}

With a flow, the situation is more delicate. The writing of a variational formulation of Galbrun's equation makes appear the following bilinear form, whose principal part does not have a fixed sign:

$$
\int_{\Omega_{b}} \operatorname{div} \boldsymbol{u} \operatorname{div} \overline{\boldsymbol{v}}-M(y)^{2} \frac{\partial \boldsymbol{u}}{\partial x} \cdot \frac{\partial \overline{\boldsymbol{v}}}{\partial x}-2 i k M(y) \frac{\partial \boldsymbol{u}}{\partial x} \cdot \overline{\boldsymbol{v}}-k^{2} \boldsymbol{u} \cdot \overline{\boldsymbol{v}} .
$$

There is no natural functional framework to formulate the problem, and moreover, no adapted finite elements (similar to the Raviart-Thomas ones for the no-flow case). On the other hand, we will be able to extend the regularization method, but it will be more delicate to perform $[14,15]$. This time the idea is to consider $\psi=\operatorname{curl} \boldsymbol{u}$ as a new unknown. Taking the curl of Galbrun's equation, we get the following relation between $\psi$ and $\boldsymbol{u}$ (where $M^{\prime}$ stands for the derivative of the Mach number with respect to $y$ ):

$$
\mathrm{D}^{2} \psi-2 M^{\prime} \mathrm{D}\left(\frac{\partial u_{x}}{\partial x}\right)=\operatorname{curl} \boldsymbol{f}(\Omega) .
$$

Note that this is a simple ordinary differential equation in $x$ (where $y$ is just a parameter). In the sequel we will show that it is then possible to calculate $\psi$ versus $\boldsymbol{u}$ and to get a regularized problem for $\boldsymbol{u}$ associated to good mathematical properties. 


\section{The treatment of the PRoblem in AN infinite DUCT}

\subsection{The dissipative problem}

First to simplify we will consider the dissipative problem where the wave number is extended to the complex plan:

$$
k_{\epsilon}=k+i \epsilon \quad \epsilon>0,
$$

and we suppose that :

$$
M(y)>0 \quad \forall y \in[0, l] .
$$

Thanks to the dissipation, we are then authorized to look for a solution with finite energy (which stands for the radiation condition). Let us consider the following problem: find $\boldsymbol{u}_{\epsilon} \in \mathrm{H}^{1}(\Omega)^{2}$ such that

$$
\begin{aligned}
& \mathrm{D}_{\epsilon}^{2} \boldsymbol{u}_{\epsilon}-\boldsymbol{\nabla}\left(\operatorname{div} \boldsymbol{u}_{\epsilon}\right)=\boldsymbol{f} \\
& \boldsymbol{u}_{\epsilon} \cdot \boldsymbol{n}=0 \quad(\partial \Omega)
\end{aligned}
$$

where

$$
\mathrm{D}_{\epsilon}=-i k_{\epsilon}+M(y) \frac{\partial}{\partial x} .
$$

The technique of regularization leads then to consider the following problem: find $\boldsymbol{u}_{\epsilon} \in \mathrm{H}^{1}(\Omega)^{2}$ and $\psi_{\epsilon} \in \mathrm{L}^{2}(\Omega)$ such that

$$
\begin{aligned}
& \mathrm{D}_{\epsilon}^{2} \boldsymbol{u}_{\epsilon}-\nabla\left(\operatorname{div} \boldsymbol{u}_{\epsilon}\right)+s \operatorname{curl}\left(\operatorname{curl} \boldsymbol{u}_{\epsilon}-\psi_{\epsilon}\right)=\boldsymbol{f} \\
& \mathrm{D}_{\epsilon}^{2} \psi_{\epsilon}-2 M^{\prime} \mathrm{D}_{\epsilon}\left(\frac{\partial u_{\epsilon, x}}{\partial x}\right)=\operatorname{curl} \boldsymbol{f} \quad(\Omega) \\
& \boldsymbol{u}_{\epsilon} \cdot \boldsymbol{n}=0 \quad \text { and } \quad \operatorname{curl} \boldsymbol{u}_{\epsilon}-\psi_{\epsilon}=0 \quad(\partial \Omega)
\end{aligned}
$$

where $s>0$ is the regularization parameter which will be fixed later.

Theorem 3.1. The second equation of (3) admits a unique solution in $\mathrm{L}^{2}(\Omega)$ :

$$
\psi_{\epsilon}=\mathbf{A}_{\epsilon} u_{\epsilon, x}+\psi_{f, \epsilon},
$$

where

$$
\psi_{f, \epsilon}=G_{\epsilon} \stackrel{x}{*} \operatorname{curl} \boldsymbol{f},
$$

and

with

$$
\mathbf{A}_{\epsilon} u_{\epsilon, x}=\tilde{G}_{\epsilon} \stackrel{x}{*} 2 M^{\prime} \frac{\partial u_{\epsilon, x}}{\partial x},
$$

$$
G_{\epsilon}(x, y)=H(x) \frac{x}{M^{2}(y)} \exp \left(\frac{i k_{\epsilon} x}{M(y)}\right),
$$

(H designs the Heaviside function), $\tilde{G}_{\epsilon}=D G_{\epsilon}=M G_{\epsilon} / x$ and $\mathbf{A}_{\epsilon}$ is continuous from $\mathrm{H}^{1}(\Omega)$ to $\mathrm{L}^{2}(\Omega)$.

Proof. The uniqueness in $\mathrm{L}^{2}(\Omega)$ is straightforward since the solutions of the homogeneous equation $D^{2} \psi_{\epsilon}=0$, which are of the form

$$
[a(y)+x b(y)] \exp \left(\frac{i k_{\epsilon} x}{M(y)}\right),
$$

cannot belong to $\mathrm{L}^{2}(\Omega)$, except if $a=0=b$. Then one can check that $G_{\epsilon} \in \mathrm{L}^{2}(\Omega)$ and satisfies for every $y \in[0, l]:$

$$
D^{2} \tilde{G}_{\epsilon}=\delta, \quad(x \in \mathbb{R}),
$$


(let us emphasise that the only $\mathrm{L}^{2}(\Omega)$ Green function is the "causal" one, vanishing upstream). The expression of $\psi_{\epsilon}$ is finally obtained by convolution of $2 M^{\prime} \mathrm{D}_{\epsilon}\left(\partial u_{\epsilon, x} / \partial x\right)+\operatorname{curl} \boldsymbol{f}$ with $G_{\epsilon}$, which gives (5) after integration by parts.

To prove that $\psi_{\epsilon} \in \mathrm{L}^{2}(\Omega)$, recall that if $h \in \mathrm{L}^{2}(\mathbb{R})$ and $g \in \mathrm{L}^{1}(\mathbb{R})$, then $h * g \in \mathrm{L}^{2}(\mathbb{R})$ and:

$$
\|h * g\|_{\mathrm{L}^{2}(\mathbb{R})} \leq\|h\|_{\mathrm{L}^{2}(\mathbb{R})}\|g\|_{\mathrm{L}^{1}(\mathbb{R})} .
$$

Since $\left\|\tilde{G}_{\epsilon}\right\|_{\mathrm{L}^{1}(\mathbb{R})}=1 / \epsilon$ we get finally:

$$
\left\|\mathbf{A}_{\epsilon} u_{\epsilon, x}\right\|_{\mathrm{L}^{2}(\Omega)} \leq \frac{2 \sqrt{S}}{\epsilon}\left\|u_{\epsilon, x}\right\|_{\mathrm{H}^{1}(\Omega)}
$$

where

$$
S=\max _{y \in[0, h]}\left[M^{\prime 2}\right]
$$

\section{Remark 3.2.}

(1) Notice that $\mathbf{A}_{\epsilon}$ has the following equivalent expression:

$$
\left(\mathbf{A}_{\epsilon} u_{\epsilon, x}\right)(x, y)=\frac{2 M^{\prime}(y)}{M(y)}\left[u_{\epsilon, x}(x, y)+\frac{i k_{\epsilon}}{M(y)} \int_{-\infty}^{x} \exp \left(\frac{i k_{\epsilon}(x-z)}{M(y)}\right) u_{\epsilon, x}(z, y) d z\right]
$$

which means that it is a zeroth order operator (continuous from $\mathrm{L}^{2}(\Omega)$ onto $\mathrm{L}^{2}(\Omega)$ ).

(2) In fact, the restriction $M(y)>0$ can be relaxed, at least for the theoretical point of view. Indeed, for a general profile, we just have to set :

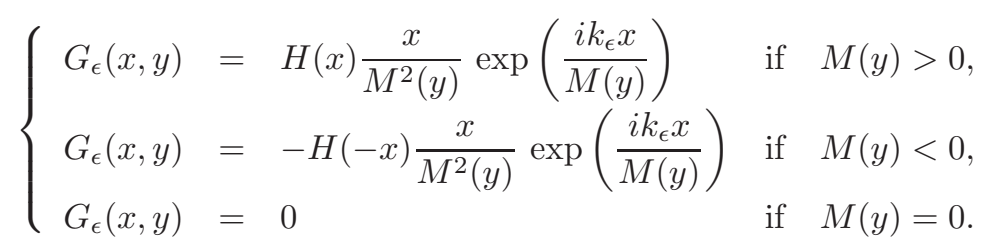

Notice in particular that contrary to formula (6), estimate (7) is not singular for vanishing Mach numbers.

However, for a practical point of view, the treatment of low Mach values is more delicate, since it requires the evaluation of highly oscillating integrals. An alternative will be suggested in the conclusion.

By injecting the expression of $\psi_{\epsilon}$ in the first equation of (3), the following problem of unknown $\boldsymbol{u}_{\epsilon}$ is finally obtained: find $\boldsymbol{u}_{\epsilon} \in \mathrm{H}^{1}(\Omega)^{2}$ solution of

$$
\left\{\begin{array}{l}
\mathrm{D}_{\epsilon}^{2} \boldsymbol{u}_{\epsilon}-\nabla\left(\operatorname{div} \boldsymbol{u}_{\epsilon}\right)+s \operatorname{curl}\left(\operatorname{curl} \boldsymbol{u}_{\epsilon}-\mathbf{A}_{\epsilon} u_{\epsilon, x}\right)=\boldsymbol{f}+\operatorname{curl} \psi_{f, \epsilon} \\
\boldsymbol{u}_{\epsilon} \cdot \boldsymbol{n}=0 \quad \text { and } \quad \operatorname{curl} \boldsymbol{u}_{\epsilon}-\mathbf{A}_{\epsilon} u_{\epsilon, x}=\psi_{f, \epsilon} \quad(\partial \Omega)
\end{array}\right.
$$

This problem has good mathematical properties: for instance, the Lax-Milgram theorem applies if $\epsilon$ is large enough. To prove this, let us first derive the variational formulation of the problem:

$$
\left\{\begin{array}{c}
\text { Find } \boldsymbol{u}_{\epsilon} \in V \text { such that } \forall \boldsymbol{v} \in V \\
a\left(\boldsymbol{u}_{\epsilon}, \boldsymbol{v}\right)=\int_{\Omega}\left(\boldsymbol{f} \cdot \overline{\boldsymbol{v}}+s \psi_{f, \epsilon} \operatorname{curl} \overline{\boldsymbol{v}}\right)
\end{array}\right.
$$


where $V=\left\{\boldsymbol{u} \in \mathrm{H}^{1}(\Omega)^{2} ; \boldsymbol{u} \cdot \boldsymbol{n}=0\right.$ on $\left.\partial \Omega\right\}$ and $a(\boldsymbol{u}, \boldsymbol{v})=b(\boldsymbol{u}, \boldsymbol{v})+c(\boldsymbol{u}, \boldsymbol{v})$ with

$$
\begin{aligned}
b(\boldsymbol{u}, \boldsymbol{v}) & =\int_{\Omega}\left(\operatorname{div} \boldsymbol{u} \operatorname{div} \overline{\boldsymbol{v}}+s \operatorname{curl} \boldsymbol{u} \operatorname{curl} \overline{\boldsymbol{v}}-M^{2} \frac{\partial \boldsymbol{u}}{\partial x} \cdot \frac{\partial \overline{\boldsymbol{v}}}{\partial x}-2 i k_{\epsilon} M \frac{\partial \boldsymbol{u}}{\partial x} \cdot \overline{\boldsymbol{v}}-k_{\epsilon}^{2} \boldsymbol{u} \cdot \overline{\boldsymbol{v}}\right), \\
c(\boldsymbol{u}, \boldsymbol{v}) & =-s \int_{\Omega} \mathbf{A}_{\epsilon} u_{x} \operatorname{curl} \overline{\boldsymbol{v}} .
\end{aligned}
$$

Theorem 3.3. The variational problem (9) is well-posed for $s>\max _{y \in[0, h]} M^{2}(y)$ and $\epsilon$ large enough.

Proof. We just need to prove that the bilinear form $a(\boldsymbol{u}, \boldsymbol{v})$ is coercive. First we note that

$$
|a(\boldsymbol{u}, \boldsymbol{u})|=\left|\frac{k_{\epsilon}}{k_{\epsilon}} a(\boldsymbol{u}, \boldsymbol{u})\right| \geq\left|k_{\epsilon}\right| \Im m\left(-\frac{a(\boldsymbol{u}, \boldsymbol{u})}{k_{\epsilon}}\right) .
$$

Concerning the bilinear form $b(\boldsymbol{u}, \boldsymbol{v})$, integrating by parts gives for all $\boldsymbol{u} \in \mathrm{H}^{1}(\Omega)^{2}$

$$
\int_{\Omega} \frac{\partial \boldsymbol{u}}{\partial x} \cdot \overline{\boldsymbol{u}}=-\int_{\Omega} \boldsymbol{u} \cdot \frac{\partial \overline{\boldsymbol{u}}}{\partial x}=-\overline{\int_{\Omega} \frac{\partial \boldsymbol{u}}{\partial x} \cdot \overline{\boldsymbol{u}}}
$$

hence $\int_{\Omega} \partial_{x} \boldsymbol{u} \cdot \overline{\boldsymbol{u}} \in i \mathbb{R}$. Thus we get that

$$
\Im m\left(-\frac{b(\boldsymbol{u}, \boldsymbol{u})}{k_{\epsilon}}\right)=\epsilon \int_{\Omega}\left[|\boldsymbol{u}|^{2}+\frac{1}{\left|k_{\epsilon}\right|^{2}}\left(|\operatorname{div} \boldsymbol{u}|^{2}+s|\operatorname{curl} \boldsymbol{u}|^{2}-M^{2}\left|\frac{\partial \boldsymbol{u}}{\partial x}\right|^{2}\right)\right] .
$$

Thanks to Costabel's identity

$$
\int_{\Omega}|\operatorname{div} \boldsymbol{u}|^{2}+|\operatorname{curl} \boldsymbol{u}|^{2}=\int_{\Omega}|\nabla \boldsymbol{u}|^{2}
$$

valid since $\boldsymbol{u} \cdot \boldsymbol{n}=0$ on $\partial \Omega$ and introducing $s_{0}=\max _{y \in[0, h]}\left[M^{2}(y)\right]<1$ we get

$$
\Im m\left(-\frac{b(\boldsymbol{u}, \boldsymbol{u})}{k_{\epsilon}}\right) \geq \epsilon \int_{\Omega}\left\{|\boldsymbol{u}|^{2}+\frac{1}{\left|k_{\epsilon}\right|^{2}}\left[\left(1-s_{0}\right)|\operatorname{div} \boldsymbol{u}|^{2}+\left(s-s_{0}\right)|\operatorname{curl} \boldsymbol{u}|^{2}\right]\right\} .
$$

Concerning the bilinear form $c(\boldsymbol{u}, \boldsymbol{v})$ we get for any $\eta>0$ :

$$
\left|\frac{c(\boldsymbol{u}, \boldsymbol{u})}{k_{\epsilon}}\right| \leq \frac{s}{2\left|k_{\epsilon}\right|} \int_{\Omega} \eta\left|\mathbf{A}_{\epsilon} u_{x}\right|^{2}+\frac{1}{\eta}|\operatorname{curl} \boldsymbol{u}|^{2}
$$

which implies, using the continuity of $\mathbf{A}_{\epsilon}$ (inequality (7))

$$
\Im m\left(-\frac{c(\boldsymbol{u}, \boldsymbol{u})}{k_{\epsilon}}\right) \geq-\frac{s}{2\left|k_{\epsilon}\right|}\left(\frac{4 S \eta}{\epsilon^{2}}\|\boldsymbol{u}\|_{\mathrm{H}^{1}(\Omega)^{2}}^{2}+\frac{1}{\eta} \int_{\Omega}|\operatorname{curl} \boldsymbol{u}|^{2}\right) .
$$

Combining the previous results we get

$$
|a(\boldsymbol{u}, \boldsymbol{u})| \geq \int_{\Omega}\left[\epsilon\left|k_{\epsilon}\right||\boldsymbol{u}|^{2}+\frac{\epsilon\left(1-s_{0}\right)}{\left|k_{\epsilon}\right|}|\operatorname{div} \boldsymbol{u}|^{2}+\left(\frac{\epsilon\left(s-s_{0}\right)}{\left|k_{\epsilon}\right|}-\frac{s}{2 \eta}\right)|\operatorname{curl} \boldsymbol{u}|^{2}-\frac{2 s S \eta}{\epsilon^{2}}\|\boldsymbol{u}\|_{\mathrm{H}^{1}(\Omega)^{2}}^{2}\right] .
$$

If we introduce the function

$$
g(\epsilon)=\min \left[\epsilon\left|k_{\epsilon}\right|, \frac{\epsilon\left(1-s_{0}\right)}{\left|k_{\epsilon}\right|}, \frac{\epsilon\left(s-s_{0}\right)}{\left|k_{\epsilon}\right|}-\frac{s}{2 \eta}\right],
$$


then $a(\boldsymbol{u}, \boldsymbol{v})$ is coercive if $g(\epsilon)>2 s S \eta / \epsilon^{2}$. Since

$$
g_{\epsilon \rightarrow \infty} \min \left[\epsilon^{2}, 1-s_{0}, s\left(1-\frac{1}{2 \eta}\right)-s_{0}\right]
$$

we deduce that if $s[1-(1 / 2 \eta)]>s_{0}$ then $g$ is positive for $\epsilon$ large enough and $a(\boldsymbol{u}, \boldsymbol{v})$ is coercive on $V \times V$.

By construction every solution of (2) belonging to $V$ is a solution of (9). The converse statement results from the following theorem:

Theorem 3.4. The solution $\boldsymbol{u}_{\epsilon}$ of problem (9) is such that curl $\boldsymbol{u}_{\epsilon}=\psi_{\epsilon}$.

Proof. Taking as test functions $\boldsymbol{v}=\operatorname{curl} \varphi$ with $\varphi \in H^{2}(\Omega) \cap H_{0}^{1}(\Omega)$ (such that $\operatorname{curl} \varphi$ belongs to $V$, in particular $v_{y}=-\partial_{x} \varphi=0$ on $\partial \Omega$ ) leads after some integrations by parts and the use of the boundary conditions of problem $(8)$ to the following orthogonality relation, where $(\cdot, \cdot)$ denotes the usual scalar product in $\mathrm{L}^{2}(\Omega)$ :

$$
\left(\operatorname{curl} \boldsymbol{u}_{\epsilon}-\psi_{\epsilon}, H_{k_{\epsilon}, M, s} \varphi\right)
$$

Here $H_{k_{\epsilon}, M, s}$ denotes the operator $\left(\mathrm{D}_{\epsilon}^{*}\right)^{2}-s \Delta$ where $\mathrm{D}_{\epsilon}^{*}=-i \overline{k_{\epsilon}}+M(y) \partial / \partial x$.

This result is easily obtained after having proved that

(1) $\forall \psi \in \mathrm{L}^{2}(\Omega)$ such that $\mathrm{D}_{\epsilon}^{2} \psi \in \mathrm{L}^{2}(\Omega)$ and $\forall \varphi \in H^{2}(\Omega) \cap H_{0}^{1}(\Omega)$,

$$
\left(\mathrm{D}_{\epsilon}^{2} \psi, \varphi\right)=\left(\psi,\left(\mathrm{D}_{\epsilon}^{*}\right)^{2} \varphi\right)
$$

(2) $\forall \boldsymbol{u} \in V$ and $\forall \varphi \in H^{2}(\Omega) \cap H_{0}^{1}(\Omega)$,

$$
b(\boldsymbol{u}, \operatorname{curl} \varphi)=\left(\operatorname{curl} \boldsymbol{u}-\mathbf{A}_{\epsilon} \boldsymbol{u},\left(\mathrm{D}_{\epsilon}^{*}\right)^{2} \varphi\right)-(\operatorname{curl} \boldsymbol{u}, s \Delta \varphi)
$$

(3) $\forall \varphi \in H^{2}(\Omega) \cap H_{0}^{1}(\Omega)$,

$$
(\boldsymbol{f}, \operatorname{curl} \varphi)_{\mathrm{L}^{2}(\Omega)^{2}}=\left(\psi_{f, \epsilon},\left(\mathrm{D}_{\epsilon}^{*}\right)^{2} \varphi\right) .
$$

Point (1) is simply an integration by parts. Point (3) is easily obtained by using point (1) after the integration by parts: $(\boldsymbol{f}, \operatorname{curl} \varphi)_{\mathrm{L}^{2}(\Omega)^{2}}=(\operatorname{curl} \boldsymbol{f}, \varphi)$, valid because $\varphi=0$ on $\partial \Omega$.

Point (2) is deduced following a similar way. $\forall \boldsymbol{u} \in H^{2}(\Omega) \cap V$ and $\boldsymbol{v} \in V$, an integration by parts leads to:

$$
b(\boldsymbol{u}, \boldsymbol{v})=\left(\mathrm{D}_{\epsilon}^{2} \boldsymbol{u}, \boldsymbol{v}\right)_{\mathrm{L}^{2}(\Omega)^{2}}+s(\operatorname{curl} \boldsymbol{u}, \operatorname{curl} \boldsymbol{v}) .
$$

Then if we take $\boldsymbol{u} \in H^{3}(\Omega) \cap V$ and $\boldsymbol{v}=\operatorname{curl} \varphi$, we can integrate $\left(\mathrm{D}_{\epsilon}^{2} \boldsymbol{u}, \operatorname{curl} \varphi\right)_{\mathrm{L}^{2}(\Omega)^{2}}$ by parts to get

$$
\left(\mathrm{D}_{\epsilon}^{2} \boldsymbol{u}, \operatorname{curl} \varphi\right)_{\mathrm{L}^{2}(\Omega)^{2}}=\left(\operatorname{curl} \mathrm{D}_{\epsilon}^{2} \boldsymbol{u}, \varphi\right)
$$

Then we have to notice that $\operatorname{curl}\left(\mathrm{D}_{\epsilon}^{2} \boldsymbol{u}\right)=\mathrm{D}_{\epsilon}^{2}(\operatorname{curl} \boldsymbol{u}-\mathbf{A} \boldsymbol{u})$ because curl $\left(\mathrm{D}_{\epsilon}^{2} \boldsymbol{u}\right)=\mathrm{D}_{\epsilon}^{2}(\operatorname{curl} \boldsymbol{u})-2 M^{\prime} \mathrm{D}_{\epsilon}\left(\partial_{x} u_{x}\right)$ and $\mathrm{D}_{\epsilon}^{2}\left(\mathbf{A}_{\epsilon} \boldsymbol{u}\right)=2 M^{\prime} \mathrm{D}_{\epsilon}\left(\partial_{x} u_{x}\right)$. Finally it is easy to conclude thanks to point (1) and to extend this result for $\boldsymbol{u} \in V$ thanks to the density of $H^{3}(\Omega) \cap V$ in $V$.

To conclude that curl $\boldsymbol{u}_{\epsilon}=\psi_{\epsilon}$ in $\mathrm{L}^{2}(\Omega)$, it suffices to show that $H_{k_{\epsilon}, M, s}$ is surjective from $\mathcal{D}\left(H_{k_{\epsilon}, M, s}\right)=$ $H^{2}(\Omega) \cap H_{0}^{1}(\Omega)$ to $\mathrm{L}^{2}(\Omega)$. Since $\Omega$ is regular (no reentrant corners), this will be achieved by using Lax-Milgram theorem after having proved the coercivity of the sesquilinear form $\left(H_{k_{\epsilon}, M, s} \varphi, \psi\right)$. For all $\varphi$ in $H_{0}^{1}(\Omega)$, we have

$$
\left(H_{k_{\epsilon}, M, s} \varphi, \varphi\right)=\int_{\Omega}\left(s|\nabla \varphi|^{2}-M^{2}\left|\frac{\partial \varphi}{\partial x}\right|^{2}-2 i \overline{k_{\epsilon}} M \bar{\varphi} \frac{\partial \varphi}{\partial x}-{\overline{k_{\epsilon}}}^{2}|\varphi|^{2}\right),
$$


which implies

$$
\Im m\left(\frac{\left(H_{k_{\epsilon}, M, s} \varphi, \varphi\right)}{\overline{k_{\epsilon}}}\right)=\epsilon \int_{\Omega}\left[|\varphi|^{2}+\frac{1}{\left|k_{\epsilon}\right|^{2}}\left(s|\nabla \varphi|^{2}-M^{2}\left|\frac{\partial \varphi}{\partial x}\right|^{2}\right)\right] .
$$

Thus $\left(H_{k_{\epsilon}, M, s} \varphi, \psi\right)$ is coercive as soon as $s>\max _{y \in[0, h]} M^{2}(y)$.

Remark 3.5. The treatment of domains with reentrant corners is an open question since, contrary to the electromagnetic case, the $H^{1}$ framework seems to be required by the presence of a mean flow $\left(\right.$ term $-M^{2} \partial_{x} \boldsymbol{u} \partial_{x} \overline{\boldsymbol{v}}$ in the variational formulation).

Corollary 3.6. For $\epsilon$ large enough, problem (2) has a unique solution in $V$.

Proof. We choose $\boldsymbol{v} \in \mathcal{D}(\Omega)^{2} \subset V$ in the variational formulation (9). Using the previous theorem and integration by parts, we obtain that the unique solution $\boldsymbol{u}_{\epsilon}$ of (9) verifies the first equation of (2) in the distributional sense. The boundary condition is also satisfied since $\boldsymbol{u}_{\epsilon} \in V$.

Remark 3.7. For a uniform flow, the well-posedness stands for every $\epsilon>0$. But for a general flow, it is not surprising that well-posedness requires sufficient absorption, to prevent exponential growing due to a possible hydrodynamic instability. We conjecture that it is possible to go further (results for small values of $\epsilon$ and limiting absorption process $\epsilon \rightarrow 0$ ) by using an explicit modal representation of the solution.

\subsection{Perfectly Matched Layers}

To develop a finite element method to compute an approximation of the outgoing solution of the initial problem (1) (problem without dissipation), Perfectly Matched layers (PML) are introduced. We face two difficulties: as in the dissipative case, the operator in Galbrun's equation is not coercive, making the finite element method unstable. Moreover the problem is set in an unbounded domain. The use of PMLs allows us to follow a similar approach as in the dissipative case. The outgoing solution is naturally selected by working in finite energy spaces. Also the coerciveness will be restored by applying a regularization technique. For practical implementation, the layers are finally truncated.

The model in the absorbing layers (the PMLs) is obtained starting from the exact model by introducing the following substitution in all the equations : $\frac{\partial}{\partial x} \longrightarrow \alpha \frac{\partial}{\partial x}$ where $\alpha$ is a complex parameter satisfying $\operatorname{Re}(\alpha)>0$ and $\operatorname{Im}(\alpha)<0$ (then the solution decreases in the layers). Like previously in the dissipative case, $\psi$ can be expressed as a fonction of $\boldsymbol{u}$ and inserted in regularized Galbrun's equation.

More precisely, layers of length $L$ are introduced on both sides of the source support. Then we solve in the domain $\left.\Omega_{L}=\right] x_{m}-L, x_{p}+L[\times] 0, l[$ described below (the physical domain around the source is defined as ]$x_{m}, x_{p}[\times] 0, l[)$

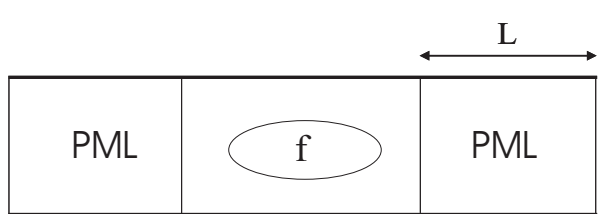

the following problem (where the index $\alpha$ means that the substitution has been introduced in the layers PML, equations are written in the sense of the distributions and we have chosen $s=1$ for the sake of simplicity):

$$
\left\{\begin{array}{l}
\mathrm{D}_{\alpha}^{2} \boldsymbol{u}-\nabla_{\alpha}\left(\operatorname{div}_{\alpha} \boldsymbol{u}\right)+\operatorname{curl}_{\alpha}\left(\operatorname{curl}_{\alpha} \boldsymbol{u}-\psi\right)=\boldsymbol{f} \quad\left(\Omega_{L}\right) \\
\psi=\mathbf{A}_{\alpha} u_{x}+\psi_{f, \alpha} \quad\left(\Omega_{L}\right) \\
\text { on } \partial \Omega_{L}:\left\{\begin{array}{l}
\boldsymbol{u} \cdot \boldsymbol{n}=0 \text { and } \operatorname{curl}_{\alpha} \boldsymbol{u}-\psi=0 \text { on } y=0 \text { and } y=l, \\
u_{x}=0=u_{y} \text { on } x=x_{m}-L \text { and } x=x_{p}+L,
\end{array}\right.
\end{array}\right.
$$

where $\mathbf{A}_{\alpha}$ denotes once again a zeroth order operator $\left(\mathbf{A}_{\alpha} u_{x}\right.$ and $\psi_{f, \alpha}$ are defined in Appendix A). 


\section{Remark 3.8.}

(1) The regularization is necessary not only for the finite element method but also for the PML method: indeed, the use of PMLs without regularization leads to a non-causal solution $\boldsymbol{u}$ [16].

(2) Here we have chosen to impose on the artificial boundaries at the vertical end of the PMLs: $\boldsymbol{u}=\mathbf{0}$. Other choices would be possible like to impose the same boundary conditions as on the horizontal boundaries: $\boldsymbol{u} \cdot \boldsymbol{n}=0$ and $\operatorname{curl}_{\alpha} \boldsymbol{u}-\psi=0$ on $\partial \Omega_{L}$. But it would produce an additional source term on the artificial boundaries, which is complicated to deal with numerically and is negligible in practice.

Tha variational formulation of problem (10) is

$$
\left\{\begin{array}{l}
\text { Find } \boldsymbol{u} \in V_{L} \text { such that } \forall \boldsymbol{v} \in V_{L} \\
a(\boldsymbol{u}, \boldsymbol{v})=\frac{1}{\alpha} \int_{\Omega_{L}}\left(\boldsymbol{f} \cdot \overline{\boldsymbol{v}}+\psi_{f, \alpha} \operatorname{curl}_{\alpha} \overline{\boldsymbol{v}}\right)
\end{array}\right.
$$

where $a(\boldsymbol{u}, \boldsymbol{v})=b(\boldsymbol{u}, \boldsymbol{v})+c(\boldsymbol{u}, \boldsymbol{v})$ with

$$
\begin{aligned}
b(\boldsymbol{u}, \boldsymbol{v}) & =\int_{\Omega_{L}}\left[\boldsymbol{u} \cdot \overline{\boldsymbol{v}}+\frac{1}{\alpha}\left(\operatorname{div}_{\alpha} \boldsymbol{u} \operatorname{div}_{\alpha} \overline{\boldsymbol{v}}+\operatorname{curl}_{\alpha} \boldsymbol{u} \operatorname{curl}_{\alpha} \overline{\boldsymbol{v}}\right)-\alpha M^{2} \frac{\partial \boldsymbol{u}}{\partial x} \cdot \frac{\partial \overline{\boldsymbol{v}}}{\partial x}\right], \\
c(\boldsymbol{u}, \boldsymbol{v}) & =-\int_{\Omega_{L}}\left(2 i k M \frac{\partial \boldsymbol{u}}{\partial x} \cdot \overline{\boldsymbol{v}}+\frac{\alpha+k^{2}}{\alpha} \boldsymbol{u} \cdot \overline{\boldsymbol{v}}+\frac{1}{\alpha} \mathbf{A}_{\alpha} u_{x} \operatorname{curl}_{\alpha} \overline{\boldsymbol{v}}\right),
\end{aligned}
$$

and where $V_{L}=\left\{\boldsymbol{u} \in \mathrm{H}^{1}\left(\Omega_{L}\right)^{2} ; \boldsymbol{u} \cdot \boldsymbol{n}=0\right.$ on $\left.\partial \Omega_{L}\right\}$.

Theorem 3.9. The variational problem (11) is of Fredholm type.

Proof. We prove that the sesquilinear form $a(\boldsymbol{u}, \boldsymbol{v})$ defines, via the Riesz representation theorem, an operator which is the sum of an isomorphism and a compact operator on $V$. The form $b(\boldsymbol{u}, \boldsymbol{v})$ is coercive on $\mathrm{V}$. Indeed, for all $\boldsymbol{u}, \boldsymbol{v} \in V$, we have

$$
\int_{\Omega_{L}} \frac{1}{\alpha}\left(\operatorname{div}_{\alpha} \boldsymbol{u} \operatorname{div}_{\alpha} \overline{\boldsymbol{u}}+\operatorname{curl}_{\alpha} \boldsymbol{u} \operatorname{curl}_{\alpha} \overline{\boldsymbol{u}}\right)=\int_{\Omega_{L}} \frac{1}{\alpha} \nabla_{\alpha} \boldsymbol{u} \cdot \nabla_{\alpha} \overline{\boldsymbol{u}}=\int_{\Omega_{L}} \alpha\left|\frac{\partial \boldsymbol{u}}{\partial x}\right|^{2}+\frac{1}{\alpha}\left|\frac{\partial \boldsymbol{u}}{\partial y}\right|^{2},
$$

which leads to

$$
\begin{aligned}
\Re e(b(\boldsymbol{u}, \boldsymbol{u})) & \geq \int_{\Omega_{L}}\left[|\boldsymbol{u}|^{2}+\Re e(\alpha)\left(1-s_{0}\right)\left|\frac{\partial \boldsymbol{u}}{\partial x}\right|^{2}+\Re e\left(\frac{1}{\alpha}\right)\left|\frac{\partial \boldsymbol{u}}{\partial y}\right|^{2}\right], \\
& \geq C_{\alpha}\|\boldsymbol{u}\|_{\mathrm{H}^{1}(\Omega)^{2}}^{2},
\end{aligned}
$$

with $C_{\alpha}=\min \left[1, \Re e(\alpha)\left(1-s_{0}\right), \Re e(1 / \alpha)\right]$.

On the other hand the form $c(\boldsymbol{u}, \boldsymbol{v})$ defines a compact operator on $V$ due to the compactness of the embedding of $\mathrm{H}^{1}\left(\Omega_{L}\right)$ into $\mathrm{L}^{2}\left(\Omega_{L}\right)$. The key point is the continuity of $\mathbf{A}_{\alpha}$ from $\mathrm{L}^{2}\left(\Omega_{L}\right)$ to $\mathrm{L}^{2}\left(\Omega_{L}\right)$. This continuity results from a formula analogous to (4) in the PML case.

Finally, if the flow is uniform, we know how to show $[16,17]$ that the solution of the problem with PML converges when the layers width $L$ tends to infinity toward the outgoing solution of the radiation problem (1), this later solution being characterized thanks to a limiting absorption technique.

Remark 3.10. Note that in the presence of the so-called upstream modes, the solution grows in the downstream layer but this does not disturb either the convergence of the solution with PML nor the quality of the calculated solution in the bounded domain $\Omega_{b}[17]$. 


\section{IMPLEMENTATION AND NUMERICAL RESUlTS}

The problem with PML is discretized thanks to Lagrange finite elements. In order to evaluate simply (by interpolation) the convolution formula in $x$, we use a structured mesh and quadrilateral-based Q2 elements, and we consider a Mach profile which does not vanish. The discretization convergence is insured since the problem is of Fredholm type.

Concerning the resolution of the linear system, it is not worth eliminating the unknown $\psi$ because the convolution operator $\mathbf{A}_{\alpha}$, which links all the freedom degrees located on the same streamline $(y=$ constant), leads to a matrix less sparse than a classical finite element matrix [15]. The unknown $\psi$ is kept and we use the following iterative algorithm initialized with $\psi^{0}=0$ :

$$
\begin{aligned}
& \mathrm{D}_{\alpha}^{2} \boldsymbol{u}^{n+1}-\nabla_{\alpha}\left(\operatorname{div}_{\alpha} \boldsymbol{u}^{n+1}\right)+\operatorname{curl}_{\alpha}\left(\operatorname{curl}_{\alpha} \boldsymbol{u}^{n+1}-\psi^{n}\right)=\boldsymbol{f} \quad\left(\Omega_{L}\right) \\
& \boldsymbol{u}^{n+1} \cdot \boldsymbol{n}=0 \quad \text { et } \quad \operatorname{curl}_{\alpha} \boldsymbol{u}^{n+1}-\psi^{n}=0 \quad\left(\partial \Omega_{L}\right) \\
& \psi^{n+1}=\mathbf{A}_{\alpha} u_{x}^{n+1}+\psi_{f} \quad\left(\Omega_{L}\right)
\end{aligned}
$$

We observe that this algorithm converges faster if the flow gradient is weaker which is not surprising since $\mathbf{A}_{\alpha}$ vanishes for a uniform flow.

The numerical method has been implemented thanks to the code MELINA [18].

The following results concern the case of an irrotational source $f$ in a duct of height $l=1$. The support of $f$ is a small disk of center $(0.5,0.5)$ and radius 0.1 and its analytical expression is :

$$
\boldsymbol{f}(x, y)=\left(\begin{array}{l}
x \\
y
\end{array}\right) \text {. }
$$

We compare the scattered field obtained for two different flow profiles, a uniform one with $M=0.5$ and a linear one given by :

$$
M(y)=0.2+0.6 y .
$$

The Mach of the uniform flow corresponds to the mean value of the linear profile.

The computational domain is represented below, with the flow profiles and the location of the source.
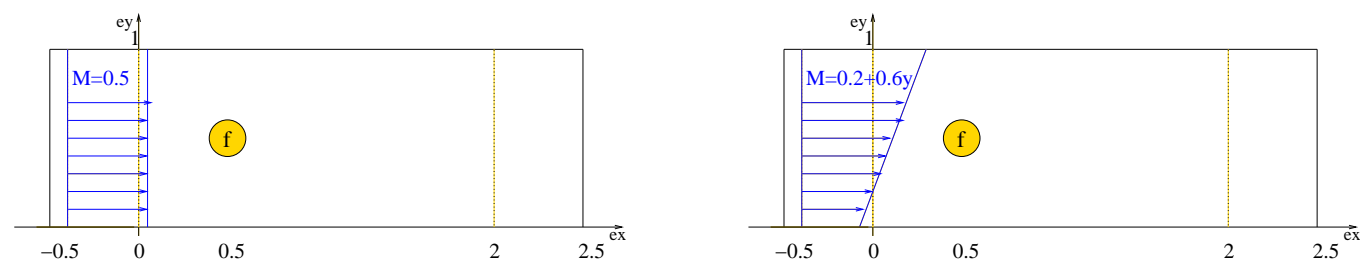

The results are obtained with 3321 degrees of freedom and $\alpha=0.5-0.5 i$.

The wavenumber $k$ is equal to 8 . At this frequency and for the uniform flow, there are 3 propagative acoustic modes in the duct.

The isovalues of the horizontal component of the displacement are represented below.

For the uniform flow, the convective effect of the flow clearly appears : the radiated acoustic field has a wavelength much larger downstream than upstream. It is essentially the third propagative mode (corresponding to a dependence with respect to $y$ of the form $\cos (2 \pi y))$ which is produced.

For the linear flow, we recognize a waveguide effect which takes the form of a concentration of the upstream acoustic field in the upper part of the duct. Downstream the source, the acoustic is hidden by the vortices, which appear as inclined lines. The source being irrotational $\left(\psi_{f}=0\right)$, it is the non-uniformity of the flow which explains the presence of vortices.

These inclined lines appear more clearly on the isovalues of $\psi$ which are plotted below : 


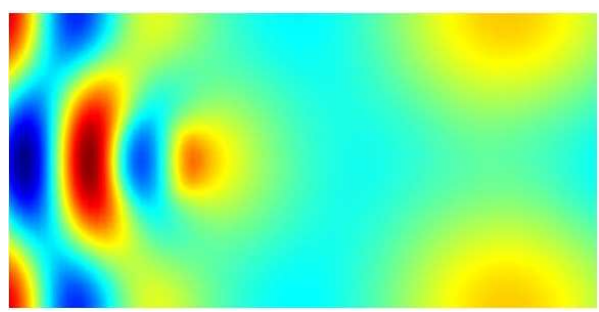

Real part of $u_{x}$, uniform flow

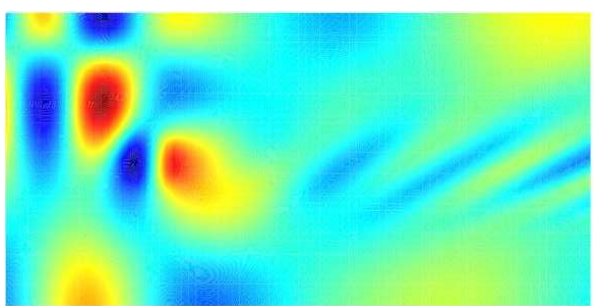

Real part of $u_{x}$, linear flow



Vorticity for the linear flow

Finally, it is interesting to observe the evolution of the solution computed during the iterative process. We give below the isovalues of the horizontal component of the displacement for the 4 first iterations. It appears that the vortices progressively take the place of the acoustic field downstream of the source.



Iteration 1



Iteration 3

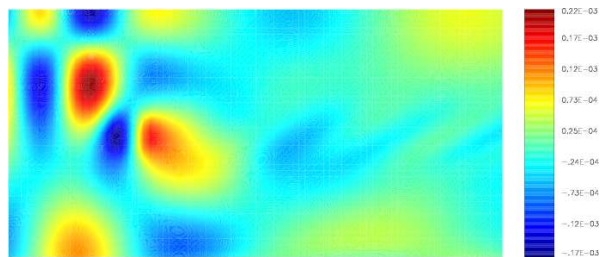

Iteration 2

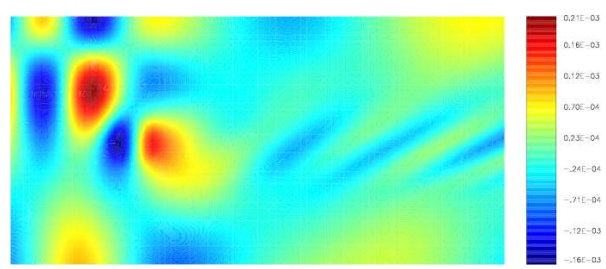

Iteration 4 


\section{Conclusion}

We have developed a numerical method allowing to calculate the field radiated by a source of perturbations in a shear flow. From a theoretical point of view, the uniform flow case has been completely studied. For a shear flow, using a dissipative process, the outgoing solution radiated by a compactly supported source has been characterized. We then proposed a variational formulation with perfectly matched absorbing layers for approximating the outgoing solution. Note that although the outgoing solution (without dissipation) is clearly defined in the uniform flow case (limiting amplitude process), we are unable to define it in the case of an arbitrary parallel flow, following the same process. However we think that for a stable flow profile the limiting amplitude process still holds.

From a numerical point of view, we developed a method based on an iterative scheme in order not to inverse non-sparse finite element matrices and we showed that we can calculate the coupling between acoustic and hydrodynamic phenomena.

The weakness of our method, in order to extend it to more practical situations (3D geometries), is the calculation of $\psi$ thanks to the convolution formula along the streamlines. Moreover, this formula degenerates when the Mach number vanishes, which happens often in practice (close to the boundaries for instance).

Currently we develop an approximated model valid for flows whose Mach number is low. This model is obtained by calculating an equivalent of the oscillating integral $\mathbf{A}_{\alpha} u_{x}$ when $M$ tends to 0 . Then we show that we can replace the integral formula

$$
\psi=\mathbf{A}_{\alpha} u_{x}
$$

by the following differential formula

$$
\psi=\frac{2 i M^{\prime}}{k} \alpha \frac{\partial u_{x}}{\partial x}
$$

whose discretization is much simpler. This model seems to be very promising for the extension to the $3 \mathrm{D}$ case.

\section{A. Definitions of $\mathbf{A}_{\alpha} u_{x}$ AND of $\psi_{f, \alpha}$}

For a physical domain defined as $] x_{m}, x_{p}[\times] 0, l[$ surrounded by infinite PMLs we get that the vorticity reads $\psi=\mathbf{A}_{\alpha} u_{x}+\psi_{f, \alpha}$ where:

$\psi_{f, \alpha}(x, y)=$

$$
\begin{aligned}
& \left(\int_{-\infty}^{x} \frac{(x-z)}{\alpha^{2} M(y)^{2}} \exp \left(i \frac{k}{\alpha M(y)}(x-z)\right) \operatorname{rot}_{\alpha} \boldsymbol{f}(z, y) d z\right. \\
& \text { if } x_{m}<x<x_{p}, \\
& \int_{-\infty}^{x_{m}} \frac{1}{\alpha M(y)^{2}}\left(x-x_{m}+\frac{x_{m}-z}{\alpha}\right) \exp \left(i \frac{k}{M(y)}\left(x-x_{m}+\frac{x_{m}-z}{\alpha}\right)\right) \operatorname{rot}_{\alpha} \boldsymbol{f}(z, y) d z \\
& +\int_{x_{m}}^{x} \frac{(x-z)}{M(y)^{2}} \exp \left(i \frac{k}{M(y)}(x-z)\right) \operatorname{rot}_{\alpha} \boldsymbol{f}(z, y) d z
\end{aligned}
$$




$$
\mathbf{A}_{\alpha} u_{x}(x, y)=\left\{\begin{array}{c}
\text { if } x<x_{m}, \\
\int_{\infty}^{x} \frac{2 M^{\prime}(y)}{\alpha M(y)} \exp \left(i \frac{k}{\alpha M(y)}(x-z)\right) \partial_{x} u_{x}(z, y) d z \\
\text { if } x_{m}<x<x_{p}, \\
\int_{-\infty}^{x_{m}} \frac{2 M^{\prime}(y)}{\alpha M(y)} \exp \left(i \frac{k}{M(y)}\left(x-x_{m}+\frac{x_{m}-z}{\alpha}\right)\right) \partial_{x} u_{x}(z, y) d z \\
\quad+\int_{x_{m}}^{x} \frac{2 M^{\prime}(y)}{M(y)} \exp \left(i \frac{k}{M(y)}(x-z)\right) \partial_{x} u_{x}(z, y) d z \\
\text { if } x_{p}<x, \quad \frac{2 M^{\prime}(y)}{\alpha M^{\prime}(y)} \exp \left(i \frac{k}{M(y)}\left(\frac{x-x_{p}}{\alpha}+x_{p}-x_{m}+\frac{x_{m}-z}{\alpha}\right)\right) \partial_{x} u_{x}(z, y) d z \\
\quad+\int_{x_{m}}^{x_{p}} \frac{2 M^{\prime}(y)}{M(y)} \exp \left(i \frac{k}{M(y)}\left(\frac{x-x_{p}}{\alpha}+x_{p}-z\right)\right) \partial_{x} u_{x}(z, y) d z \\
\quad+\int_{x_{p}}^{x_{m}} \frac{2 M^{\prime}(y)}{\alpha M(y)} \exp \left(i \frac{k}{M(y)}\left(\frac{x-z}{\alpha}\right)\right) \partial_{x} u_{x}(z, y) d z
\end{array}\right.
$$

Thanks to these formulas it is straightforward to prove that $\psi \in \mathrm{L}^{2}(\mathbb{R} \times] 0, l[)$.

\section{REFERENCES}

[1] S. Duprey, Etude mathématique et numérique de la propagation acoustique d'un turboréacteur, Thèse de Doctorat de l'Université Henry Poincaré-Nancy 1 (2006).

[2] J. P. Coyette, Manuel théorique ACTRAN, Free Field Technologies, Louvain-la-Neuve, Belgique (2001).

[3] J. Diaz, And P. Joly, Robust high order non-conforming finite element formulation for time domain fluid-structure interaction, J. Comput. Acoust., 13 (2005), pp. 403-431.

[4] C. Bogey, C. Bailly, and D. Juve, Computation of Flow Noise Using Source Terms in Linearized Euler's Equations, AIAA Journal, 40 (2002), pp. 235-243.

[5] C. Bailly, And D. Juve, Numerical Solution of Acoustic Propagation Problems Using Linearized Euler Equations, AIAA Journal, 38 (2000), pp. 22-29.

[6] T. Hagstrom, I. Nazarov, and N. Peake, Absorbing Layers and radiation Conditions for Jet Flow Simulations, 8th AIAA/CEAS Aeroacoustics Conference, Breckenridge, CO, USA, June 2002.

[7] F. Q. Hu, A Stable, Perfectly Matched Layer for Linearized Euler Equations in Unsplit Physical Variables, J. Comput. Acoust., 173 (2001), pp. 455-480.

[8] C. K. W. Tam, L. Auriault, and F. Cambuli, Perfectly Matched Layer as an Absorbing Boundary Condition for the Linearized Euler Equations in Open and Ducted Domains, J. Comput. Acoust., 144 (1998), pp. 213-234.

[9] M. Bernacki, S. Lanteri, and S. Piperno, Time-Domain Parallel Simulation of Heterogeneous Wave Propagation on Unstructured Grids Using Explicit, Nondiffusive, Discontinuous Galerkin Methods, J. Comput. Acoust., 14 (2006), pp. 57-82.

[10] P. Delorme, P. Mazet, C. Peyret, and Y. Ventribout, Computational Aeroacoustics applications based on a discontinuous Galerkin method, Comptes rendus de mécanique, 333 (2005), pp. 676-682.

[11] H. Galbrun, Propagation d'une onde sonore dans l'atmosphère terrestre et théorie des zones de silence, Gauthier-Villars, Paris, France (1931).

[12] G. Gabard, R. J. Astley, and M. B. Tahar, Stability and accuracy of finite element methods for flow acoustics: II. Two-dimensional effects, International Journal for Numerical Methods in Engineering, 63 (2005), pp. 974-987.

[13] F. Treyssede, G. Gabard, and M. B. Tahar, A mixed finite element method for acoustic wave propagation in moving fluids based on an Eulerian-Lagrangian description, JASA, 113 (2003), pp. 705-716.

[14] A.-S. Bonnet-Ben Dhia, G. Legendre, and E. Lunéville, Analyse mathématique de l'équation de Galbrun en écoulement uniforme, Compte-Rendu à l'Académie des Sciences, Tome 329, Série II b (2001), pp. 601-606.

[15] A.-S. Bonnet-Ben Dhia, Eve-Marie Duclairoir, G. Legendre and J.-F. Mercier, Time-harmonic acoustic propagation in the presence of a shear flow, Journal of Computational and Applied Mathematics, to appear.

[16] E. Bécache, A.-S. Bonnet-Ben Dhia and G. Legendre, Perfectly matched layers for time-harmonic acoustics in the presence of a uniform flow, SIAM Journal on Numerical Analysis, to appear. 
[17] E. Bécache, A.-S. Bonnet-Ben Dhia and G. Legendre, Perfectly matched layers for the convected Helmholtz equation, SIAM Journal on Numerical Analysis, Volume 42, Number 1 (2004), pp. 409-433.

[18] D. Martin, On line documentation of MÉLINA, http://perso.univ-rennes1.fr/daniel.martin/melina/www/homepage.html. 